\title{
Detection and molecular characterization of Slovak tomato isolates belonging to two recombinant strains of potato virus $Y$
}

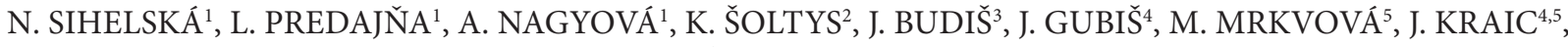 \\ D. MIHÁLIK ${ }^{5,6}$, M. GLASA ${ }^{1 *}$
}

\begin{abstract}
${ }^{1}$ Institute of Virology, Biomedical Research Centre, Slovak Academy of Sciences, Dúbravská cesta 9, 84505 Bratislava, Slovak Republic; ${ }^{2}$ Comenius University Science Park, Comenius University in Bratislava, Ilkovičova 8, 841 04, Bratislava, Slovak Republic; ${ }^{3}$ Department of Computer Science, Faculty of Mathematics, Physics and Informatics, Comenius University in Bratislava, Mlynská dolina F1, 84248 Slovak Republic; ${ }^{4}$ National Agriculture and Food Centre-Research Institute of Plant Production, Bratislavská cesta 122, 92168 Pieštany, Slovak Republic; ${ }^{5}$ Department of Biotechnologies, Faculty of Natural Sciences, University of SS. Cyril and Methodius, J. Herdu 2, 91701

Trnava, Slovakia; ' ${ }^{2}$ stitute of High Mountain Biology, University of Žilina, Univerzitná 8215/1, 01026 Žilina, Slovak Republic
\end{abstract}

Summary. - Total RNAs from a symptomless tomato plant were subjected to next-generation sequencing (NGS) analysis, revealing the presence of a single viral agent - potato virus Y (PVY). The analysis of determined full-length genome sequence assigned the PVY SL16 isolate to the recombinant PVY-N-Wi strain group. A series of primers targeting the four main recombinant junction (RJ) sites were used for characterization of additional 5 tomato PVY isolates recovered in Western Slovakia. Based on the partial sequences, the isolates could be classified as belonging to PVY-N-Wi and PVY-NTNa strain groups. Interestingly, both these distinct recombinant PVY types were identified in mixed infection in one tomato sample (SL31). Our results further reinforce the data on the complexity of PVY infection and confirm the recombination as a significant evolutionary process shaping the PVY diversity.

Keywords: potyvirus; Solanum lycopersicum; detection; recombination; NGS

\section{Introduction}

PVY is a worldwide viral pathogen of economically important crops such as potato, pepper, tobacco and tomato. The virus is effectively transmitted by vegetatively propagated material, such as tubers, and by aphids in a non-persistent manner, making its control difficult (Scholthof et al., 2011; Karasev and Gray, 2013).

Tomatoes (Solanum lycopersicum) are one of the most economically important vegetables. The symptoms of PVY

*Corresponding author. E-mail: Miroslav.Glasa@savba.sk; phone: +421-2-59302446.

Abbreviations: $\mathrm{PVY}=$ potato virus $\mathrm{Y} ; \mathrm{RJ}=$ recombinant junction; NGS = next-generation sequencing; DAS-ELISA = double antibody sandwich ELISA; ORF = open reading frame; $p i p o=$ pretty interest ing Potyviridae ORF infection on this crop may include crinkling of the young leaves, necrotic mottling, mosaics and often interveinal yellow spots (Abad and Jordá, 2000).

The study of genetic diversity and the evolutionary mechanisms is important to understand virus epidemiology and emergence and is a prerequisite to design effective diagnostic tools, and implement effective disease management decisions. PVY is known to have a huge inter- and intra-strain diversity (Karasev and Gray, 2013). Moreover, besides mutations, recombination is another evolutionary process shaping the PVY genetic structure and possibly increasing its adaptability and emergence (Karasev et al., 2011). Such complex variability may complicate the correct identification of isolates especially using serological tests (Gugerli and Fries, 1983; Tian et al., 2014).

Traditionally, there are two main PVY biological groups commonly recognized according to the symptomatology they induce on potato and tobacco carrying a series of re- 
sistance genes, i.e. the ordinary (PVY-O) and tobacco veinal necrosis (PVY-N) strain, together with three other biological groups - PVY-C, PVY-E, and PVY-Z (Jones, 1990; Singh et al., 2008; Karasev et al., 2011; Karasev and Gray, 2013). Recombination between PVY strain groups has given rise to new strain groups, e.g. PVY-NTN, PVY-N-Wi, PVY-N:O, having the tendency to increase worldwide (Glais et al., 2002; Lorenzen et al., 2006a; Karasev and Gray, 2013). Further molecular approaches have made it possible to characterize and classify PVY strains based on genome characteristics (Lorenzen et al., 2006b; Chikh et al., 2010), however, sometimes without a clear-cut linking to their biological traits (Karasev and Gray, 2013).

In recent years, the molecular research of viruses was accelerated by NGS approaches, providing high speed and high throughput, enabling powerful analysis of the plant "virome" and pathogen characterization without a priori knowledge (Massart et al., 2014).

Although PVY belongs to the best studied potyviruses, data on the genetic diversity of PVY were not yet reported from Slovakia. In this work, the complete and partial sequence data of PVY isolates originated from tomato were determined, demonstrating the presence of two recombinant PVY strains in Slovakia.

\section{Materials and Methods}

NGS analysis and determination of full-length PVY SL16 sequence. A tomato plant cv. Tornado F1 (referred further as SL16), showing no obvious viral-like symptoms, was originally grown in a private garden in Cífer, Western Slovakia (GPS: $48^{\circ} 19.001^{\prime} \mathrm{N}$, $\left.17^{\circ} 28.918^{\prime} \mathrm{E}\right)$.

Total RNAs designated for NGS analysis were extracted using the Nucleo Spin RNA Plant kit (Macherey-Nagel, Duren, Germany) from top leaves of original tomato plant (collected in July 2015) in order to avoid the accumulation of mutations during transfer to and propagation in experimental hosts. To remove the unwanted ribosomal RNA, the Ribo-Zero rRNA Removal Kit (Illumina, San Diego, USA) was applied. A depleted total RNA sample was used for ds cDNA reverse-transcription with SuperScript II (Thermo Fisher Scientific, Waltham, USA) kit.

The cDNA sample was purified using the DNA Clean \& Concentrator $^{\text {riw }}-5$ - DNA kit (Zymo Research, Irvine, USA), fluorometrically quantified with the Qubit 2.0 Fluorometer (Thermo Fisher Scientific, Waltham, USA) and processed with the Illumina transposon-based chemistry (Nextera XT, Illumina, San Diego, USA). A 12-cycle PCR enabled paired-end indexing of the sample. Fragment size selection with 1,8x sample volume of Agencourt AMPure XP magnetic beads (Beckman Coulter, Brea, CA, USA) was carried out. The molarity of the final library was assessed according to library fragment size distribution using the Agilent 2100 Bioanalyzer (Agilent Technologies, Santa Clara, USA) and its quantity determined by a Qubit 2.0 Fluorometer. Finally, the indexed denatured and diluted library was sequenced with 150bp paired-end sequencing. The primary analysis including image analysis, base calling and data quality assessment was performed on an Illumina NextSeq benchtop sequencer.

Low quality read ends were trimmed using a Trimmomatic (Bolger et al., 2014). Trimmed reads were aligned to the genome of S. lycopersicum (GenBank Acc. No. AEKE00000000.2) with the Bowtie2 mapper (Langmead et al., 2012). All reads with concordant match to the genome were filtered out and the remaining reads aligned to viral genomes database (ftp://ftp.ncbi.nih.gov/genomes/ Viruses/all.fna.tar.gz), downloaded on February 2016.

Secondary data analysis was performed by de novo assembly using a CLC Genomics Workbench 7.5 (https://www.qiagenbioinformatics.com/) with altered parameters (Mismatch cost 2, Insertion cost 3, Deletion cost 3, Length fraction 0,7 and Similarity fraction 0,9 ) and minimum contig length $1000 \mathrm{bp}$. All contigs were subsequently mapped to the reference genome of the host (AEKE00000000.2) in CLC v7.5 with default parameters and the unmapped contigs were aligned against the virus BLAST database (Altschul et al., 1990).

The full-length genome sequence was completed by RT-PCR using a specific primerPVY9566F (5'-TCGTTGTTGTGGATGATTC3-3') in combination with oligo $d(\mathrm{~T})$ for determination of the 3 'end. The 5 'end (nts 1-325) was amplified using PVY5-F 5' -ATTAAAACAACTCAATACAAC-3' and PVY325R 5' TAAGTTGTGTTTCCAGATC- 3 '. The accuracy of the NGS data was also checked by sequencing of the genome portions encompassing the RJs amplified by specific primers (Fig. 1).

Molecular characterization of $P V Y$ isolates. The 45 tomato plants randomly selected from 4 distinct gardens, i.e. Bzince pod Javorinou (1 sample), Cífer (12 samples), Čachtice (4 samples), Pezinok (28 samples) were tested for the presence of PVY by DAS-ELISA using commercial antibodies (Loewe Biochemica, Sauerlach, Germany).

PVY-positive samples were further analyzed by RT-PCR. To amplify various genomic regions, a two-step RT-PCR protocol was used. The first strand cDNA was synthesized by reverse transcription of total RNA using pdN6 random primers and the Avian myeloblastosis virus (AMV) reverse transcriptase (Promega Corp., Madison, WI, USA), with the view to use the same RT preparation for all individual PCRs. Several pairs of primers were designed based on available complete genome sequences retrieved from the GenBank ( $n=150$, accessed on September 2015) in order to amplify parts of the P1, HC/P3, 6K2/VPg and CP/3'UTR regions (Fig. 1). All PCR amplifications were performed using the proofreading TaKaRa Ex Taq ${ }^{\text {Tax }}$ polymerase (Takara Bio Inc., Shiga, Japan). The PCR products were purified using the Wizard PCR Preps DNA Purification System (Promega Corp.) and directly sequenced by priming the sequencing reaction with the same oligonucleotides as used for PCR. The sequences were analyzed by Blast in order to find the closest relative.

Complete and partial sequences of PVY used for comparison were retrieved from the GenBank (www.ncbi.nlm.nih.gov). Sequence and 


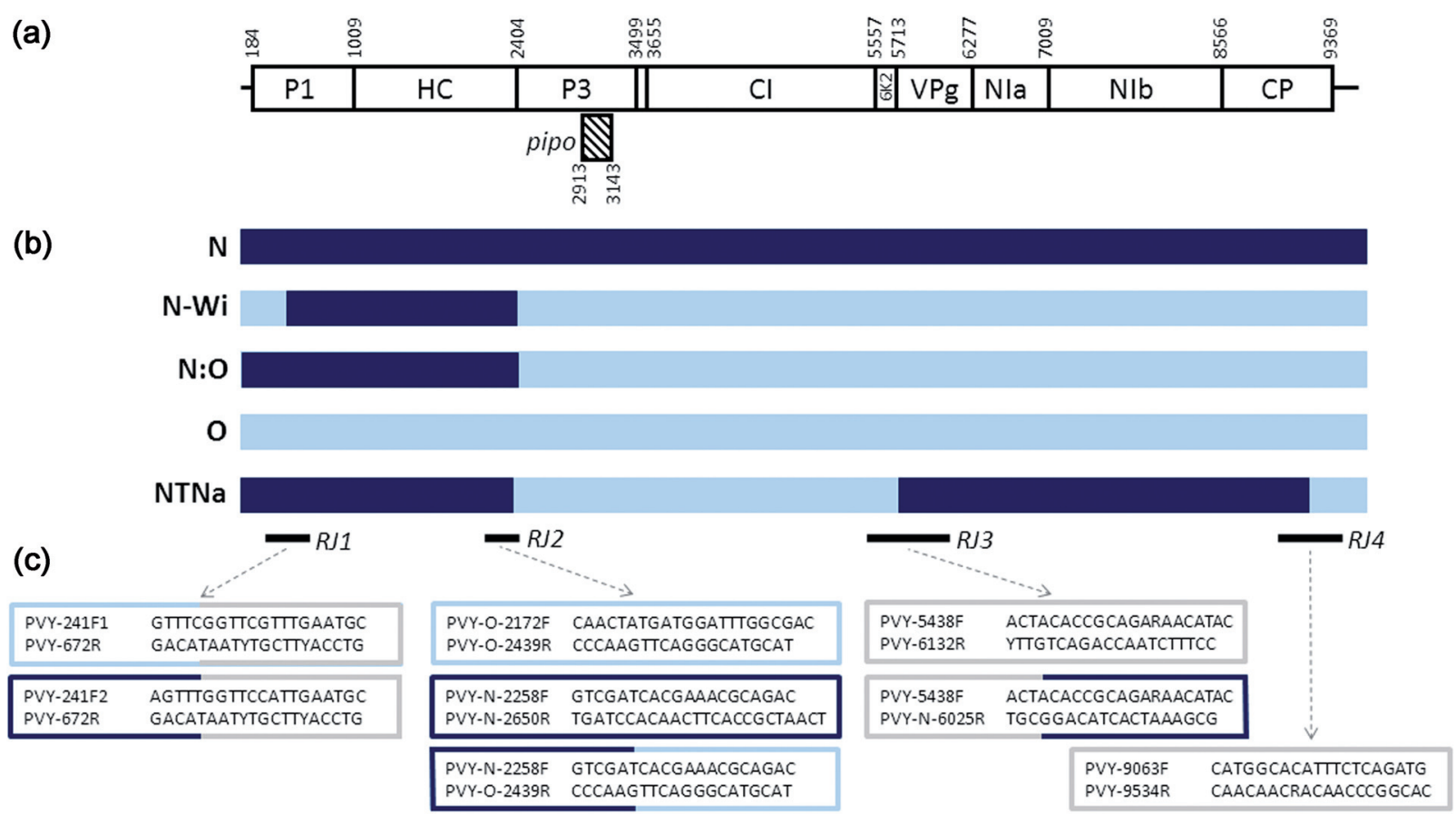

Fig. 1

(a) Schematic representation of the PVY-SL16 genome showing the nucleotide positions delimiting the respective potyviral functional products. (b) Presentation of selected types of recombinant PVY genomes based on Karasev and Gray (2013)

Parental sequences are colour-differentiated (PVY-0: azure, PVY-N: dark blue). The bars correspond to the products amplified by RT-PCR, encompassing four main recombinant junction (RJ) sites. (c) Position and sequences of primers used for RT-PCR. Polyvalent primers are in the grey frame, while strain-specific primers are in colour frames. The primers targeting RJ1, RJ3 and RJ4 were designed in this work, those spanning the RJ2 are from Lorenzen et al. (2006b).

phylogenetic analyses and comparisons were performed using the MEGA v.6 (Tamura et al., 2013). Searches for potential recombination events in complete PVY genome were performed using the RDP4 v.4.63 software (Martin et al., 2015) using default settings.

Biological test. The SL16 isolate was mechanically transmitted from original tomato host to tomato cv. Monalbo and Mobaci and Nicotiana benthamiana grown under controlled conditions (14 hr light/10 hr dark photoperiod, $55 \mu \mathrm{mol} \mathrm{m} \mathrm{m}^{-2} \mathrm{~s}^{-1}$ photon flux density, day/night temperature: $25 / 18^{\circ} \mathrm{C}$ ). Development of symptoms was evaluated visually and the virus presence regularly checked by DAS-ELISA.

\section{Results and Discussion}

The NGS analysis revealed the presence of a single viral agent in the symptomless tomato plant

As symptom expression did not always predict infection status, the occurrence of symptomless viral infections of plants represents a potential threat for subsequent uncontrolled dissemination of viruses, especially those transmit- ted by vectors, such as PVY. Therefore, the total RNA were isolated from an asymptomatic tomato SL16 and further analyzed by NGS to reveal possible virus presence.

For the first screening of the SL16 tomato sample, from a total of 25,312,514 reads, a filtered dataset of trimmed reads $(23,479,316)$ were mapped against the reference sequence of S. lycopersicum AEKE00000000.2. Of the 3,712,478 remaining reads that were aligned with Bowtie 2 mapper to the virus database, 322,764 were mapped. Within the analysed reads a single viral species, Potato virus $Y$, was identified. In total, 272,063 reads $(96.03 \%$ of all reads identified within the virus database) were mapped to GenBank complete genome sequence of Potato virus Y (NC_001616.1) with 93\% identity. Data analysis did not confirm the presence of other viral agents in this sample.

In the additional analysis, of 25,312,514 reads, a total of $8,349,210$ reads with average length 120.90 bp were assembled into 3,831 contigs. The total contig length represented $6,286,601$ nucleotides. The consensus length of contigs differed from 953 to 13,831 with mean contig length 1,640 bp. The quality of assembled contigs was determined by $\mathrm{N} 50=1.331$ (statistic measuring assembly quality). The 
average coverage varied from 2.84 to $166,076.76$. Of 3,831 contigs, 2,685 were mapped to the reference tomato genome. An alignment by using BLAST revealed that within the remaining 1,146 contigs a single viral contig identified as Potato virus $Y$ is present. This contig, 9,647bp in size and with average coverage 4,429.16x, tightly mapped to the genome of PVY isolate IUNG-12 (JF927760.1).

The fact that PVY-SL16 was isolated from an asymptomatic tomato plant further pinpoints the problem of symptomless, latent or cryptic infections (Richert-Pöggeler and Minarovits, 2014) since these apparently uninfected plants can serve as an effective source of inoculum for further virus spread. Environmental conditions, genetic factors or plant of the molecular variability of virus may play a role in symptom development (Simmons et al., 2013). As ascertained by experimental mechanical inoculation of tomato plants (Monalbo and Mobaci), despite the positive DAS-ELISA, the absence of symptoms was observed also on these genotypes, although a pronounced symptoms (chlorotic mottling, leaf puckering and deformations) were noted in inoculated $N$. benthamiana plants.

Analysis of the complete genome sequence shows SL16 to be a PVY N-Wi type

The determined complete genome of the PVY-SL16 isolate was composed of 9,697 nucleotides, excluding the 3 'terminal poly (A) tail. Based on complete genome comparison, SL16 was found to be most closely related to the PVY N-Wi isolate from Poland, isolated from tobacco (JF927760) with 99.5\% identity at the nucleotide level (99.8\% at amino acid level). Very high identity was also noted with other N-Wi isolates from Poland (JF927751, JF927753, JF927758, all originated from tobacco), from Brazil (JQ924286, JQ924288, both from potato) and Israel (KX184816, isolated from potato). The grouping of SL16 was further confirmed by phylogenetic analysis (Fig. 2). Similarly to other N-Wi isolates, two recombination breakpoints in the SL16 genome were located at the central part of $\mathrm{P} 1$ and close to the $\mathrm{HC}-\mathrm{pro} / \mathrm{P} 3$ region (around nt positions 488 and 2394) based on the RDP4 analysis (data not shown).

The SL16 polyprotein sequence (3061 aa) was strictly collinear with that of other PVY N-Wi isolates. The sequences of nine cleavage sites identified within a large ORF agreed with consensus sequences of other PVY-N-Wi isolates (data not shown). All characteristic motifs of potyviral proteins fundamental for viral cycle life and vector transmission were conserved in the PVY SL16 genome. Computer analysis of the SL16 polyprotein (with a calculated molecular mass of 347.292) showed that the CCC and PTK motifs, associated with aphid transmission (Huet et al., 1994), were conserved in the HC-Pro at aa positions 574-576 and 592-594, respectively. The highly conserved potyviral tetrapeptid KITC, which is also involved in aphid transmission of potyviruses, is located on the N-terminal domain of HC-Pro

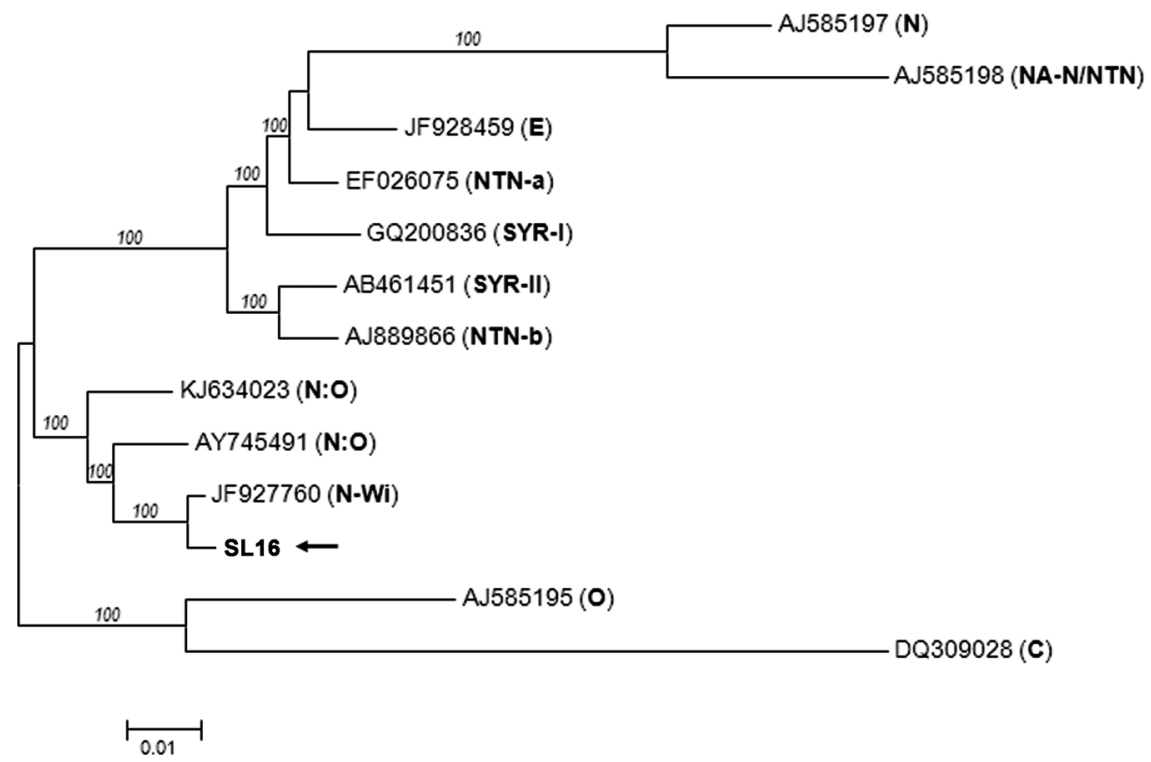

Fig. 2

Phylogenetic tree of representative PVY isolates reconstructed using the complete nucleotide sequences

The sequences used for analysis were recovered from the GenBank database and are identified by their Acc. No. and strain group affiliation. The scale bar represents a distance of 0.01 substitutions per site. Bootstrap values (1,000 bootstrap resamplings) are indicated on the branches. SL16 isolate is highlighted by an arrow. 
(aa 334-337). Metal binding motif FRNK, affecting the symptom expression (Gal-On and Raccah, 2000), has been found at aa position 463-466. As expected, the CI protein, thought to function as a RNA helicase in genome replication, contained the expected NTP binding motif GAVGSGKST (aa 1242-1250) and other motifs characteristic of helicase proteins (Kadare and Haenni, 1997): VLLIEPTRPL (aa 1262-1271), KVSAT (aa 1358-1362), LVYV (aa 1409-1412), VATNIIENGVTL (aa 1460-1471) and GERIQRLGRVGR (aa 1504-1515). Typical conserved motifs of potyviral polymerases QPSTVVDN and GDD (Domier et al., 1987), found in the NIb protein at aa positions 2587-2594 and 2626-2628, respectively. The DAG tripeptide, involved in the process of aphid transmission (Atreya et al., 1990), was present in the N-terminus of capsid protein at the positions 2800-2802. The predicted molecular mass of CP was 17, 233.

An additional coding ORF, termed pipo, that overlaps the P3-encoding region of the polyprotein ORF in the $-1 /+$ 2 reading frame (Chung et al., 2008) was found located at nucleotides 2913-3143, encoding 76 amino acids. Its highly conserved G1-2A6-7 motif was present as G2A7.

Although the molecular variability of PVY was studied in the neighboring central-European countries (Moravec et al., 2003; Hasiów-Jaroszewska et al., 2015), to our knowledge, this is the first complete PVY sequence from Slovakia (deposited to the GenBank database Acc. No. KX713170).

\section{Detection of PVY-NTNa and N-Wi isolates on tomato}

Additional 5 naturally PVY-infected tomato plants were detected in September 2015 using DAS-ELISA from three distinct locations in Western Slovakia (Bzince pod Javorinou, Cífer, Čachtice). No PVY infection was detected among 28 tested tomatoes from the locality Pezinok. Similarly to the SL16 plant, no obvious viral symptoms could be recorded in these PVY-infected plants.
Although a huge genetic diversity was observed for PVY with a number of unique recombination events described recently, basically nine main recombination types are recognized (Karasev and Gray, 2013), most of them having PVY and $\mathrm{PVY}^{\mathrm{N}}$ as parental sequences. Recombinant types have one to four relatively conserved recombinant junction (RJ) sites, allowing to design molecular tools for strain differentiation (e.g. Lorenzen et al., 2006b; Chikh et al., 2010). Due to the molecular characteristics, however, some RJ did not distinguish between molecular strains groups (e.g. N-Wi/ $\mathrm{NTN} / \mathrm{N}: \mathrm{O}$ in the RJ2 or O/N:O/N-Wi in the RJ4, see Fig. 1) requiring a complex $\mathrm{RJ}$ comparison, instead of a single gene/ genome portion analysis.

In this work, a series of primers spanning the $4 \mathrm{RJ}$ were used to analyze and type the PVY isolates (Fig. 1). The BLAST analysis of sequences obtained from each PCR products allowed to determine their closest relatives and assign them into molecular groups. No non-recombinant strain isolates was found among 6 PVY isolates characterized. Based on this approach, two isolates, SL32 and SL33, were unambiguously classified as NTNa, while SL36 and SL39 were shown to belong to the $\mathrm{N}-\mathrm{Wi}$ strain (Table 1). Interestingly, two molecularly distinct PVY types, N-Wi and NTNa, were identified in the sample SL31 as ascertained by type-specific PCR of RJ1 and RJ3. The heterologous nature of potyviral populations and mix infection of two or more isolates within a sample was reported (Lecoq et al., 2011; Abad and Jordá, 2000). Our results thus further reinforce the data on the complexity of potyviral infection.

Natural populations of RNA viruses rapidly generate genetic diversity because of a combination of high mutation rates, rapid replication, and large population sizes (Desbiez et al., 2011). Also, recombination amongst PVY genome sequences is likely to have significant evolutionary implications for the virus, as a dramatic incidence of new recombinant strains occurred in recent years (Lorenzen et al., 2006a; Ka-

Table 1. PVY isolates characterised in this study and their characteristics

\begin{tabular}{|c|c|c|c|c|c|}
\hline Isolate & Locality & $\begin{array}{l}\text { RJ1 } \\
229-639(411 \text { bp) } \\
\text { 5'P1 }\end{array}$ & $\begin{array}{l}\text { RJ2 } \\
2274-2413(140 \mathrm{bp}) \\
3^{\prime} \mathrm{HC}\end{array}$ & $\begin{array}{l}\text { RJ3 } \\
5444-6097(654 \mathrm{bp} / 556 \mathrm{bp}) \\
6 \mathrm{~K} 2-\mathrm{VPg}\end{array}$ & $\begin{array}{l}\text { RJ4 } \\
\text { 9062-9554 (493 bp) } \\
\text { CP-3'UTR }\end{array}$ \\
\hline SL31 & Čachtice & $\begin{array}{l}\text { N-Wi }(\text { KX713184) } \\
\text { NTNa }(\text { KX713185) }\end{array}$ & $\begin{array}{l}\text { NTN/N:O/N-Wi (KX752780) } \\
\text { n.d. }\end{array}$ & $\begin{array}{l}\text { O/N:O/N-Wi (KX713179)a } \\
\text { NTN (KX713177) }\end{array}$ & $\begin{array}{l}\text { O/N:O/N-Wi (KX713172) } \\
\text { n.d. }\end{array}$ \\
\hline SL32 & Bzince pod Javorinou & NTN/N:O (KX713186) & NTN/N:O/N-Wi (KX752777) & NTN (KX713180) & NTN (KX713173) \\
\hline SL33 & Bzince pod Javorinou & NTN/N:O (KX713187) & NTN/N:O/N-Wi (KX752778) & NTN (KX713181) & NTN (KX713174) \\
\hline SL36 & Cífer & N-Wi (KX713182) & NTN/N:O/N-Wi (KX752776) & O/N:O/N-Wi (KX713176) & $\mathrm{O} / \mathrm{N}: \mathrm{O} / \mathrm{N}-\mathrm{Wi}(\mathrm{KX} 713171)$ \\
\hline SL39 & Cífer & N-Wi (KX713183) & NTN/N:O/N-Wi (KX752779) & O/N:O/N-Wi (KX713178) & $\mathrm{O} / \mathrm{N}: \mathrm{O} / \mathrm{N}-\mathrm{Wi}(\mathrm{KX} 713173)$ \\
\hline SL16 & Cífer & N-Wi (KX713170) & NTN/N:O/N-Wi (KX713170) & O/N:O/N-Wi (KX713170) & $\mathrm{O} / \mathrm{N}: \mathrm{O} / \mathrm{N}-\mathrm{Wi}(\mathrm{KX} 713170)$ \\
\hline
\end{tabular}

The analysed sequences are identified by their nt position (numbered based on the reference JF927760 full length sequence), length and genome part targeted. Primer sequences were removed prior to the analysis. The assignment of sequence to the PVY molecular strain groups was determined by BLAST. The sequences were deposited to GenBank Acc. Nos. given into brackets. n.d. = not determined; $\mathrm{a}, \mathrm{b}=$ two sequence variants identified in a single sample (see text). 
rasev and Gray, 2013). In this work, two recombinant types of PVY have been detected on tomatoes in Slovakia, in one case, in double mixed infection.

As it was shown for other potyviruses, whose genetic diversity is affected by recombination, the characterization or typing methods focusing solely on single genome region should provide erroneous results (Glasa et al., 2004). In case of PVY, the partial sequencing and mutual analysis of the genome parts encompassing the four main RJ in the PVY genome proved useful to assign the isolates into molecular strain groups.

Acknowledgements. This work was supported by grants APVV14-0055 and APVV-0380-12 from the Slovak Research and Development Agency and partially by the grant REVOGENE (ITMS 26240220067) supported by the Research \& Development Operational Programme funded by the ERDF.

\section{References}

Altschul SF, Gish W, Miller W, Myers EW, Lipman DJ (1990): Basic local alignment search tool. J. Mol. Biol. 215, 403-410. https:/doi.org/10.1016/S0022-2836(05)80360-2

Abad P, Jordá C (2000): Characterization of potato Y potyvirus isolates from tomato crops in Islas Canarias (Spain). EPPO Bull. 30, 281-287. https:/doi.org/10.1111/j.13652338.2000.tb00896.x

Atreya CD, Raccah B, Pirone TP (1990): A point mutation in the coat protein abolishes aphid transmissibility of a potyvirus. Virology 178, 161-165. https:/doi.org/10.1016/00426822(90)90389-9

Bolger AM, Lohse M, Usadel B (2014): Trimmomatic: a flexible trimmer for Illumina sequence data. Bioinformatics 30, 2114-2120. https:/doi.org/10.1093/bioinformatics/ btu170

Chikh Ali M, Maoka T, Natsuaki KT, Natsuaki T (2010): The simultaneous differentiation of Potato virus $\mathrm{Y}$ strains including the newly described strain PVY(NTN-NW) by multiplex PCR assay. J. Virol. Methods 165, 15-20. https:/ doi.org/10.1016/i.jviromet.2009.12.010

Chung BY, Miller WA, Atkins JF, Firth AE (2008): An overlapping essential gene in the Potyviridae. Proc. Natl. Acad. Sci. USA 105, 5897-5902. https:/doi.org/10.1073/ pnas. 0800468105

Desbiez C, Moury B, Lecoq H (2011): The hallmarks of "green" viruses: Do plant viruses evolve differently from the others? Infect. Gen. Evol. 11, 812-824. https:/doi.org/10.1016/j. meegid.2011.02.020

Domier LL, Shaw JG, Rhoads RE (1987): Potyviral proteins share amino acid sequence homology with picorna-, como-, and caulimoviral proteins. Virology 158, 20-27. https:/ doi.org/10.1016/0042-6822(87)90233-9

Gal-On A, Raccah B (2000): A point mutation in the FRNK motif of the potyvirus HC-Pro gene alters the symptom expression in cucurbits and exhibits protection against severe homologous virus. Phytopathology 90, 467-473. https:/ doi.org/10.1094/PHYTO.2000.90.5.467

Glais L, Tribodet M, Kerlan C (2002): Genomic variability in potato Potyvirus Y (PVY): Evidence that PVYNW and PVYNTN variants are single to multiple recombinants between PVYO and PVYN isolates. Arch. Virol. 147, 363-378. https:/doi.org/10.1007/s705-002-8325-0

Glasa M, Palkovics L, Komínek P, Labonne G, Pittnerová S, Kúdela O, Candresse T, Šubr Z (2004): Geographically and temporally distant natural recombinant isolates of Plum pox virus are genetically very similar and form a unique PPV subgroup. J. Gen. Virol. 85, 2671-2681. https:/doi. org/10.1099/vir.0.80206-0

Gugerli P, Fries P (1983): Characterization of monoclonal antibodies to potato virus $\mathrm{Y}$ and their use for virus detection. J. Gen. Virol. 64, 2471-2477. https:/doi.org/10.1099/0022$\underline{1317-64-11-2471}$

Hasiów-Jaroszewska B, Stachecka J, Minicka J, Sowinski M, Borodynko N (2015): Variability of Potato virus Y in tomato crops in Poland and development of a reverse-transcription loop-mediated isothermal amplification method for virus detection. Phytopathology 105, 1270-1276. https:/ doi.org/10.1094/PHYTO-08-14-0219-R

Huet H, Gal-On A, Meir E, Lecoq H, Raccah B (1994): Mutations in the helper component protease gene of Zucchini yellow mosaic virus affect its ability to mediate aphid transmissibility. J. Gen. Virol. 75, 1407-1414. https:/doi. org/10.1099/0022-1317-75-6-1407

Jones RAC (1990): Strain group specific and virus specific hypersensitive reactions to infection with potyviruses in potato cultivars. Ann. Appl. Biol. 117, 93-105. https:/doi. org/10.1111/j.1744-7348.1990.tb04198.x

Kadare G, Haenni AL (1997): Virus-encoded RNA helicases. J. Virol. 71, 2583-2590.

Karasev AV, Hu X, Brown CJ, Kerlan C, Nikolaeva OV, Crosslin JM, Gray SM (2011): Genetic diversity of the ordinary strain of Potato virus Y (PVY) and origin of recombinant PVY strains. Phytopathology 101, 778-785. https:/doi. org/10.1094/PHYTO-10-10-0284

Karasev AV, Gray SM (2013): Continuous and emerging challenges of Potato virus Y in potato. Annu. Rev. Phytopathol. 51, 571-586. https:/doi.org/10.1146/annurev-phyto-082712$\underline{102332}$

Langmead B, Salzberg SL (2012): Fast gapped-read alignment with Bowtie 2. Nature Methods 9, 357-359. https:/doi. org/10.1038/nmeth.1923

Lecoq H, Fabre F, Joannon B, Wipf-Scheibel C, Chandeysson C, Schoeny A, Desbiez C (2011): Search for factors involved in the rapid shift in Watermelon mosaic virus (WMV) populations in South-eastern France. Virus Res. 159, 115-123. https:/doi.org/10.1016/j.virusres.2011.05.004

Lorenzen JH, Meacham T, Berger PH, Shiel PJ, Crosslin JM, Hamm PB, Kopp H (2006a): Whole genome characterization of Potato Virus Y isolates collected in the western USA and their comparison to isolates from Europe and Canada. Arch. Virol. 151, 1055-1074. https:/doi.org/10.1007/ s00705-005-0707-6 
Lorenzen JH, Piche, LM, Gudmestad NC, Meacham T, Shiel P (2006b): A multiplex PCR assay to characterize Potato virus $\mathrm{Y}$ isolates and identify strain mixtures. Plant Dis. 90, 935-940. https:/doi.org/10.1094/PD-90-0935

Massart S, Olmos A, Jijakli H, Candresse T (2014): Current impact and future directions of high throughput sequencing in plant virus diagnostics. Virus Res. 188, 90-96. https:/doi. org/10.1016/j.virusres.2014.03.029

Martin DP, Murrell B, Golden M, Khoosal A, Muhire B (2015): RDP4: Detection and analysis of recombination patterns in virus genomes. Virus Evol. 1, vev003. https:/doi.org/10.1093/ ve/vev003

Moravec T, Cerovská N, Boonham N (2003): The detection of recombinant, tuber necrosing isolates of Potato virus Y (PVY(NTN)) using a three-primer PCR based in the coat protein gene. J. Virol. Methods 109, 63-68. https:/ doi.org/10.1016/S0166-0934(03)00047-8

Richert-Pöggeler KR, Minarovits J (2014): Diversity of latent plantvirus interactions and their impact on the virosphere. In Gaur RK, Hohn T, Sharma P (Eds): Plant Virus-Host Interaction. Academic Press, Elsevier, San Diego, pp. 263-275. https:/doi.org/10.1016/b978-0-12-411584$\underline{2.00014-7}$
Scholthof KB, Adkins S, Czosnek H, Palukaitis P, Jacquot E, Hohn T, Hohn B, Saunders K, Candresse T, Ahlquist P, Hemenway C, Foster GD (2011): Top 10 plant viruses in molecular plant pathology. Mol. Plant Pathol. 12, 938-954. https:/ doi.org/10.1111/j.1364-3703.2011.00752.x

Simmons HE, Dunham JP, Zinn KE, Munkvold GP, Holmes EC, Stephenson AG (2013): Zucchini yellow mosaic virus (ZYMV, Potyvirus): Vertical transmission, seed infection and cryptic infections. Virus Res. 176, 259-264. https:/ doi.org/10.1016/j.virusres.2013.06.016

Singh RP, Valkonen JPT, Gray SM, Boonham N, Jones RAC, Kerlan C, Schubert J (2008): Discussion paper: The naming of Potato virus Y strains infecting potato. Arch. Virol. 153, 1-13. https:/doi.org/10.1007/s00705-007-1059-1

Tamura K, Stecher G, Peterson D, Filipski A, Kumar S (2013): MEGA6: Molecular Evolutionary Genetics Analysis version 6.0. Mol. Biol. Evol. 30, 2725-2729. https:/doi. org $/ 10.1093 / \mathrm{molbev} / \mathrm{mst} 197$

Tian Y-P, Hepojoki J, Ranki H, Lankinen H, Valkonen JPT (2014): Analysis of Potato virus Y coat protein epitopes recognized by three commercial monoclonal antibodies. PLoS ONE 9, e115766. https:/doi.org/10.1371/journal. pone. 0115766 